\title{
Corrigendum
}

\section{Effect of coherence of generating radiations on optical parametric up-conversion} [Opt. Quant. Elect. 11 (1979) 287-295]

The description of nonlinear optical processes in terms of photon fluxes (photon numbers), used in the above paper, that was originally based on the imagination of mutual interaction of monochromatic waves, has rather limited application. Namely, the equations for photon fluxes (Equations 8-14) [derived from the equations for complex amplitudes (Equation 4)] that describe the nonlinear coupling between individual waves, do not involve the description of development of coherence of interacting waves. This description does not involve the second order correlations of the type $\left\langle A_{i}\left(\zeta_{1}\right) A_{i}^{*}\left(\zeta_{2}\right)\right\rangle$ describing the interference effects, and therefore, in general, such a description does not correctly describe the nonlinear process for arbitrary coherent states of generating radiations. Moreover, the conservation laws for radiations with arbitrary coherence are correct for average photon numbers only.

The description used describes satisfactorily the sum-frequency generation for coherent radiations and for chaotic quasi-monochromatic radiations with a very high degree of coherence if the relation $1 / \Gamma_{i} \gg z_{0}(i=1,2)$ holds $\left(\Gamma_{i}\right.$ being the spatial Lorentzian spectral half-width and $z_{0}$ denotes the thickness of a nonlinear medium).

When using the iteration method for solving the amplitude equations [1], we get in the first approximation the following expression for the average photon flux of the sum-frequency wave at $\omega_{3}$ which is generated by two chaotic waves at $\omega_{1}$ and $\omega_{2}$ having the defined spectral half-widths $\Gamma_{1}$ and $\Gamma_{2}$ :

$$
\begin{aligned}
\left\langle N_{3}(z)\right\rangle & =\mu^{2} N_{1,0} N_{2,0} \int_{0}^{z} \mathrm{~d} \zeta_{1} \int_{0}^{z} \mathrm{~d} \zeta_{2} \exp \left[-\left(\Gamma_{1}+\Gamma_{2}\right)\left|\zeta_{1}-\zeta_{2}\right|\right] \\
& =\frac{\mu^{2} N_{1,0} N_{2,0}}{\left(\Gamma_{1}+\Gamma_{2}\right)}\left(2 z-\frac{2}{\left(\Gamma_{1}+\Gamma_{2}\right)}\left\{1-\exp \left[-\left(\Gamma_{1}+\Gamma_{2}\right) z\right]\right\}\right),
\end{aligned}
$$

Where the following factorization relation for chaotic generating radiations having a Lorentzian shape of spectral line was used $[2,3]$ :

$$
\left\langle A_{i}\left(\zeta_{1}\right) A_{i}^{*}\left(\zeta_{2}\right)\right\rangle=\left(\frac{\mu_{0}}{\epsilon_{0}}\right)^{1 / 2} \frac{\left\langle I_{i}\right\rangle}{n_{i}} \exp \left(-\Gamma_{i}\left|\zeta_{2}-\zeta_{1}\right|\right) \quad(i=1,2) .
$$

Evidently, for greater $\Gamma_{i}$ the efficiency of sum-frequency generation decreases with decreasing degree of coherence of the generating radiations (with increasing $\Gamma_{i}$ ) which contradicts the previous incomplete results. Thus the curves in Fig. 1 for values $\eta_{1} \gtrsim 1$ and also the Formulae (33) are not correct.

\section{References}

1. S. A. AKHAMANOV and A. S. CZIR KIN, 'Statisticzeskie Yavleniya v Nelineynoy Optike' (Izdatelstvo Moskovskogo Universiteta, Moscow, 1971).

2. J. PEŘINA, 'Coherence of Light' (Van Nostrand Reinhold Co., London, 1972).

3. R. LOUDON, 'The Quantum Theory of Light' (Clarendon Press, Oxford, 1973).

Received 2 October 1979

P. CHMELA

Laboratory of Optics Palacky University

Leninova 26

77146 Olomouc

Czechoslovakia 